\title{
Comparative Value of a Sorting Procedure and Quantitative Descriptive Analysis to Investigate the Influence of Processing Parameters: Case Study of Hydrolysate Production From Salmon By-Products
}

\author{
Mireille Cardinal ${ }^{1, *}$, Régis Baron ${ }^{1}$, Christelle Kouakou ${ }^{1,2,4}$, Carole Prost ${ }^{2,4}$ and Philippe Courcoux ${ }^{3,4}$
}

\author{
${ }^{1}$ Laboratory of Science and Technology of Marine Biomass, IFREMER, Nantes, France \\ 2 Nantes-Atlantic College of Veterinary Medicine and Food Science, ONIRIS, Nantes, France \\ ${ }^{3}$ USC "Sensometrics and Chemometrics Laboratory", ONIRIS, Nantes, France \\ ${ }^{4}$ LUNAM University Nantes Angers Le Mans, Nantes, France \\ *: Corresponding author : Mireille Cardinal, tel. +33-2-40-37-40-61; fax +33-2-40-37-40-71; \\ email address : cardinal@ifremer.fr
}

\begin{abstract}
:
Many papers have recently discussed the value of a free sorting method as a rapid and simple alternative to quantitative descriptive analysis, considered the reference tool for food sensorial characterization. The aim of the present paper is to evaluate whether this method of free sorting can also be used to investigate the influence of processing parameters. An experimental design was applied to production conditions of enzymatic hydrolysates from salmon by-products. The effect of four processing parameters (time and temperature of hydrolysis, sugar and antioxidant addition) on the odor of the hydrolysates was studied using a sorting task with 45 untrained panelists and a quantitative descriptive analysis carried out with 11 trained panelists. This study on 21 enzymatic hydrolysates confirms the similarity of the two sensory maps and shows the value of free sorting in the sensory characteristic description step, especially to avoid missing some descriptors. It also highlights in this example that a holistic approach as sorting can reveal more easily than profiling the significant effects of process parameters on sensory characteristics and the relationships between sensory dimensions and instrumental measurements of volatile compounds.
\end{abstract}

\section{Practical Applications}

Having a rapid and simple method to evaluate the sensory properties of food products and to investigate the effect of processing parameters could be useful during product development steps. Results from the present case study showed that compared with quantitative and descriptive analysis, the holistic approach of sorting task could clearly relate sensory characteristics to processing parameters and seemed efficient for industrial applications and product development. 


\section{Introduction}

Recent studies have highlighted the value of the sorting technique in the sensory field to assess the similarities of a set of products easily and quickly. This procedure, relatively old and well known in psychology and medical fields (Wild et al. 1965, Morton 1969, Rosch 1973), has seen a renewal of interest for its potential applications in sensory evaluation. The task consists of asking assessors, trained or not, to group products according to their sensory similarities or differences. This technique has been applied to a large range of products and sensory characteristics, from food products such as cheese (Lawless et al. 1995), jellies (Tang and Heymann 2002), yogurts (Saint-Eve et al. 2004), beers (Lelièvre et al. 2008)], and virgin olive oils (Santosa et al. 2010) to non-food products such as fabrics or plastic parts for automobiles (Giboreau et al. 2001, Faye et al. 2004) and also recently to link perceptual experience to textural terms (Varela et al. 2013). The procedure is generally completed by a verbal description of each identified group which leads to a perceptual map based on the dimensions of multidimensional scaling (MDS), the typical analysis performed with sorting data, although alternative approaches have been proposed recently (Abdi et al. 2007, Cadoret et al. 2009). Free sorting has been completed by a hierarchical structure called taxonomic free sorting (Courcoux et al. 2011), which allows a distance to be assigned between groups enabling better discrimination. The value of the sorting technique is also illustrated by a proposed method to test the stability of a sorting map (Blancher et al. 2012). Previous studies suggested that this technique used by naïve consumers could give the same sensory maps as those produced by trained sensory panels (Faye et al. 2006, Cartier et al. 2006, Veramendi et al., 2013). It could therefore offer an alternative to quantitative descriptive analysis, the method widely used in sensory analysis by research and industry to obtain a detailed description of a product in terms of descriptors and intensities (Stone et al 1974) in order to optimise processes or find relationships with consumer preferences. Sorting by untrained panellists appears to be a time-saving alternative to quantitative descriptive analysis for rapid sensory mapping, as it does not require a long stage of panellist selection and training while still producing consistent product maps (Varela and Ares 2012).

The aim of this study is to analyse the efficiency of a sorting task to investigate the influence of processing parameters on the production of enzymatic hydrolysates from salmon byproducts. The effect of four processing parameters (time and temperature of hydrolysis, sugar and antioxidant addition) on the odour of the hydrolysates was studied and results from the sorting task were compared with those from a quantitative descriptive analysis. Volatile compounds generated during the processing were also analysed in each hydrolysate and the relationship with both sensory maps was studied to give a complementary point of view on data for the comparison.

\section{Materials and methods}

\subsection{Samples}

\subsubsection{Preparation}

Samples used for this study came from a research project on the production of enzymatic hydrolysates from salmon by-products. This project aimed to investigate the effect of hydrolysis conditions on several quality parameters (Kouakou et al. 2013). Sensory characteristics have been identified as a key factor for further applications in the food industry and were therefore studied more specifically as well as the associated volatile compounds. Samples were prepared from salmon by-products (heads and frames) obtained from a local smoked salmon company (Piriac, France). After this raw material was ground, an enzyme 
Protamex (Novozymes, Bagsvaerd, Denmark) was added to the mince $(0.15 \% \mathrm{w} / \mathrm{w})$ under different processing conditions. After hydrolysis, all biological reactions were stopped by heating at $95^{\circ} \mathrm{C}$ for $30 \mathrm{~min}$ and samples were centrifuged at $9800 \mathrm{~g}$ at $15^{\circ} \mathrm{C}$ for $30 \mathrm{~min}$. For each processing condition, the aqueous phases collected were separated and sampled into two 100-ml plastic flasks for further sensory evaluation, quantitative descriptive analysis and free sorting. All samples were stored at $-80^{\circ} \mathrm{C}$ until evaluation.

\subsubsection{Experimental design}

An experimental design, based on the Doehlert design completed by some specific experiments, was performed to study the effect of four different independent variables; temperature (from $30^{\circ} \mathrm{C}$ to $60^{\circ} \mathrm{C}$ ) and time of hydrolysis (from $30 \mathrm{~min}$ to $470 \mathrm{~min}$ ), addition of sugar (xylose, industrial grade, provided by Danisco, Surrey, United Kingdom) or natural antioxidant (a commercial mixture of natural tocopherols and rosemary from the company Jan Dekker International, Wormerveer, the Netherlands) to the mince. This required the preparation of forty samples (Kouakou et al. 2013). A supplementary sample was introduced as a control in sessions of descriptive and quantitative analysis. This control sample was prepared without enzyme at a temperature of $60^{\circ} \mathrm{C}$, with a process time of $360 \mathrm{~min}$, without sugar and antioxidant. Thus, 41 samples were finally obtained.

It seemed difficult to carry out a sorting task on such a large number so a selection of a sample sub-set, representative of the entire set of hydrolysates, was compiled. A sorting task on odours of around twenty samples was considered achievable. The selection was based on the $D$-optimality criterion, which consists in selecting the 20 products from the 40 candidates such that det $\left(\left(X^{T} X\right)^{-1}\right)$ is minimal. Linear, quadratic and first order interaction terms for the four processing variables were used to compute the $X$ matrix of experiments. This criterion is equivalent to minimising the generalised variance of the estimator (Atkinson and Donev 1992). In order to achieve this selection according to the $D$-optimality criterion, an iterative procedure based on the Fedorov exchange algorithm (Fedorov 1972) was used. The control sample was also added so 21 samples were finally presented for the sorting task (Table 1).

\subsection{Sensory evaluation conditions}

The two sensory methods were performed in the same conditions, in individual partitioned booths controlled for temperature $\left(20^{\circ} \mathrm{C}\right)$ and light (day light, $\mathrm{T}=6500^{\circ} \mathrm{K}$ ). For the descriptive and quantitative method, data were collected with a computerised system (Fizz, Biosystèmes, Dijon, France). The day before the sensory test, samples were thawed overnight at $2^{\circ} \mathrm{C}$. Then, the possible difference in colour between samples was masked with a black colouring agent, neutral in smell. About $8 \mathrm{ml}$ of each hydrolysate was poured into a polystyrene crystal flask, assigned a 3 digit-number and kept at $18^{\circ} \mathrm{C}$ before the test.

\subsection{Sensory methods}

Two experiments were performed (1) a free sorting with forty-five untrained panellists, (2) a quantitative descriptive analysis with eleven trained panellists.

\subsubsection{Sorting technique Untrained panellists}

The panel was recruited from staff and students of the two research organisations involved in the project, 19 from Ifremer and 26 from Oniris; these 45 people were panellists untrained on hydrolysate products and had no previous experience of this product. However, they could be qualified as initiated in sensory evaluation because they sometimes take part in food tests. Taking into account the results of previous studies, which showed that the stability of a sorting map can be influenced by the complexity of the task and which recommended at least 
25 people (Faye et al. 2006) or to start a work with 30 evaluations (Cartier et al. 2006), the number of 45 panellists seemed a reasonable figure for this study.

Free sorting procedure

Panellists received the 21 samples simultaneously in a random order. Previous studies concluded that it was possible to sort until 20 beers (Cholet et al., 2011) and that olfactory fatigue did not affect the results from sorting task with 16 perfumes (Veramendi et al., 2013), it is the reason why we suggested to present 21 samples in order to have a good representativeness of the product space while avoiding a too heavy task for panellists. They were asked to sort the products into groups based on odour similarities. They had to make at least two groups and no more than twenty. Panellists had all the time necessary to perform the task and were required to smell fresh air when necessary. Once performed the sorting, panellists could verify the proximity of samples within each group after a resting time. Then, a description of the odour characteristics was required for each group. Panellists could use their own vocabulary and suggest one or several words to describe each group. No glossary was presented.

\subsubsection{Quantitative descriptive analysis Trained panellists}

Samples were sniffed by a trained panel of 11 people, 8 females and 3 males, selected from members of the internal panel of Ifremer. These panellists have regular training in odour perception and characterisation of seafood products and were selected according to their sensory performances. During the specific training step, individual performances were checked at a multidimensional level with a comparison of samples discrimination results with the discrimination of the group and for each attribute, the consistency in product ranking was evaluated in comparison with the result of the group. Finally 11 out of the 16 panellists who started the study were selected for the final evaluation. The descriptor selection and panellist training are described by Kouakou et al. (2013).

\section{Sensory procedure}

A quantitative descriptive analysis (ISO 13299 2003) was performed to quantify nine selected descriptors of odour: global intensity, marine, fat fish, dried fish, roasted, rancid, potato, sulphur and brine fish. In order to evaluate the 41 samples, panellists were required to attend seven sessions of profiling, two per week, in a comparative way. An experimental design was constructed in order to balance for contrast effects. Four parameters were balanced; hydrolysis temperature and time, presence of sugar and antioxidant. Six hydrolysates including the control sample were presented in each session. The intensity of each sensory descriptor was directly scored on an unstructured scale anchored by the terms low intensity (0) and high intensity (10) using data acquisition software. With the aim of comparing the two sensory methods, only the data from the 21 samples used for free sorting were analysed.

\subsubsection{Comparison of configurations}

A global index of proximity of the two factorial configurations, the RV coefficient (Robert and Escoufier 1976), was computed on three dimensions.

\subsection{Volatile compound analysis}

2.4.1. Extraction of the volatile compounds by Headspace Solid Phase MicroExtraction (HS-SPME)

$5 \mathrm{ml}$ of hydrolysate was placed in a 20-mL glass vial closed with a screw top equipped with a Teflon septum. The sample was equilibrated for $60 \mathrm{~min}$ at $40^{\circ} \mathrm{C}$. The extraction of the volatile 
compounds was performed with a Carboxen/PDMS fibre $(85 \mu \mathrm{m}, 1 \mathrm{~cm}$, Carboxen/PDMS StableFlex, Supelco, Sigma-Aldrich Chimie, Lyon, France) for $15 \mathrm{~min}$ at $40^{\circ} \mathrm{C}$. Analyses sample.

\subsubsection{Gas chromatography / Mass spectrometry / FID}

The apparatus used was a gas chromatograph (Agilent 7890A, Wilmington, DE, USA) equipped with a flame ionisation detector (FID) and coupled to a mass spectrometer (electronic impact source, Agilent 5975CNetwork, Wilmington, DE, USA). The inlet temperature was $260^{\circ} \mathrm{C}$, the FID detector temperature $250^{\circ} \mathrm{C}$ and the MS detector temperature $280^{\circ} \mathrm{C}$. The carrier gas was helium and the pressure was $62.4 \mathrm{kPa}$. The splitless mode was used for the injection, and the desorption time was $3 \mathrm{~min}$. The capillary column was a DBWAX (30 m, $0.25 \mathrm{~mm}, 0.5 \mu \mathrm{m}$, J\&W Scientific, Folsom, CA). The program used was $40^{\circ} \mathrm{C}$ for $10 \mathrm{~min}$, ramp to $120^{\circ} \mathrm{C}$ at $4^{\circ} \mathrm{C} / \mathrm{min}$, ramp to $240^{\circ} \mathrm{C}$ at $20^{\circ} \mathrm{C} / \mathrm{min}$ then equilibrium at $240^{\circ} \mathrm{C}$ for 5 min. Effluent from the end of the GC was split 1/1 between the MS and the FID. Peaks were integrated with MSD Chemstation software (Agilent Technologies). Mass spectra were recorded in electron impact mode $(70 \mathrm{eV})$ between 33 and $300 \mathrm{~m} / \mathrm{z}$ mass range at a scan rate of 2.7 scan.s-1.

The volatile compounds were identified according to 3 criteria: comparison of their Kovats retention index with the literature, comparison of their mass spectra with those of the Wiley 6 library and injection of the corresponding standards. The semi-quantified results were obtained from the FID chromatogram and expressed in peak area. The repeatability of the method was $9 \%$.

\subsection{Data Analysis \\ 2.5.1. Sensory data analysis Sorting data}

From the sorting task, a measure of dissimilarity between two stimuli was considered as the number of subjects who separated these two items into different groups (Faye et al. 2004). This dissimilarity matrix was submitted to a Multidimensional Scaling technique (MDS) which provided a factorial configuration of the stimuli and exhibited the main sensory dimensions of the set of products. A non-metric procedure was used, considering that dissimilarities have only an ordinal interpretation (Borg and Groenen 2005). In order to assess the stability of the resulting configuration and to evaluate whether products were perceived as significantly different from a sensory point of view, confidence ellipses were built using a bootstrapping approach according to the procedure described by Courcoux et al. (2011). Cadoret and Husson (2013) showed that ellipses built by a method based on total bootstrap can be interpreted as confidence areas. The volumes of ellipses inform on sensory distances between samples but also on variability between panellist evaluations.

To analyse the sensory characteristics of each product, the terms used for one group were associated with each product of the group. A general matrix (products $x$ terms) with the number of occurrences of each term for describing each product was generated from the entire panel. Then, the terms with the same meaning were grouped together by the panel leader and those that appeared less than three times for one product were removed from the final matrix. Correlations between each term and each MDS dimension were computed in order to provide an interpretation of the underlying dimensions.

Profiling data

Sensory data were submitted to two-way analysis of variance (ANOVA) with products and panellists as independent factors in order to identify significant product effects and descriptors 
involved in this discrimination. Significant differences between means were determined using Duncan's multiple range test $(\mathrm{p}<0.05)$. A principal component analysis (PCA) without descriptor using XLSTAT for Windows version 2012 (Addinsoft, Paris, France). As for the sorting data, a procedure of total bootstrapping was applied to set up the confidence ellipses.

\subsubsection{Relation between volatile compounds and sensory data}

After an identification step, the volatile compounds were grouped according to their probable origin (lipid oxidation, Maillard reactions, fermentation, marine environment, other origin) or according to their main chemical structure (hydrocarbon, alcohols, aldehydes, ketones, acids, furans, sulphurs, pyridine and thiazol). For the two types of classification, the sum of the peak areas for each volatile compound gathered in each group (origin or structure) was calculated. Global matrices (products x volatile compound groups) were obtained. Correlations were calculated between each volatile compound group and each dimension of the product configuration for the sorting task and profiling procedures

\subsubsection{Effect of process parameters on sensory properties of hydrolysates}

The effect of process parameters on sensory dimensions was assessed by means of ordinary least squares regression (OLSR). For the two sensory procedures (sorting task and quantitative descriptive analysis), the three sensory dimensions were regressed on the factors of the experimental design, using a quadratic model. The resulting regression coefficients are interpreted as the quantification of the main effects of factors, interactions between these factors, and quadratic effects. As the process parameters are not expressed in the same units, the regression coefficients are not directly comparable so the t-values were computed to check the significance of these coefficients. An absolute value of t higher than 2.5 indicates a significant effect of the parameter at the 0.05 level of significance. These t-values were represented in order to compare the contributions of the effects of factors on the different sensory dimensions.

\section{Results}

\subsection{Comparison of the sensory map from free sorting and descriptive and quantitative analysis}

Free sorting results

The first plane of the MDS applied on free sorting data represented $81.2 \%$ of the total inertia. The first axis showed a clear discrimination between two main groups of products (Fig. 1a). One of these groups was constituted of twelve products, with the highest coordinates along the first axis. The dimension 2 allowed the separation of three different groups; one group with samples 39, 38 and 20, another including samples 35, 37 and 40 and a specific group with the sample number 3. Fifteen attributes were used to describe these groups. The correlation of these attributes with the MDS dimensions enabled an interpretation of the main sample characteristics (Fig. 1b). On the first dimension, criteria of roasted, brine, cooked and fat fish were associated with the group of twelve products and, at the opposite, seaweed, lean fish, crustacean and sulphur for a second group of products. The attribute "cheese odour" had the highest correlation with dimension 2 and explained some differences between samples. On the first dimension, sample 3, for example, showed a roasted characteristic like the other samples of this group but the position on the second dimension (negative coordinate) indicated at the same time a distinct characteristic, namely a cheese odour in this sample. This specific odour was probably the reason for the separation from the other "roasted" samples. Samples with a negative coordinate on the first dimension presented a larger distribution of location along the second dimension. From the top to the bottom, samples were associated 
with marine and crustacean characteristics and, to a lesser extent, chemical (samples 38 and 39) and to sulphur, seaweed and spoilage odour for samples located on the bottom left side of the figure (35, 37 and 40). The third dimension (not presented) added some information in terms of sample discrimination and allowed a separation of samples 20 and 41 from the others. The terms most frequently associated with these two products were the same as those observed in dimension 2; marine, crustacean and also chemical. However, a detailed study of the description data (Table 2) showed that, compared to samples 38 and 39 on one hand and to samples 35,37 and 40 on the other hand, the frequencies of attributes quotations used for these two products were different. For example, a marine odour was more frequently described in samples 20 and 41 than in sample 40, while the sulphur odour in contrast was less often noticed compared to samples 35,37 and 40 but more than in sample 38 . The fat fish descriptor was also used with different frequencies, more often than for samples 35 and 37 and less than for sample 38 .

Descriptive and quantitative analysis results

Data from profiling were submitted to a two-way analysis of variance with panellists and products as independent factors. This treatment identified significant differences between products and descriptors with the most discriminative power. Comparison of $F$ values for the product effect showed that roasted odour had the highest followed, in decreasing order, by sulphur, global intensity, fat fish, dried fish, potato, marine, rancid and odour of fish in brine. The first plane of the unstandardised principal component analysis (PCA) accounted for $85.3 \%$ of the total information (Fig. 2a). The first axis $(75.1 \%$ of total inertia) was mainly created by the criteria roasted, dried fish, global intensity, marine and fat fish while sulphur, fat fish and marine odours were mostly involved in the creation of the second component (10.25\% of the inertia) (Fig. 2b). A clear discrimination between samples appeared on this plane. Along axis 1, two groups of products were separated, according to the global intensity of the odour as well as the intensity of roasted and dried notes. As in the sorting procedure, a group of nine samples was found on one hand and a group of twelve samples on the other hand. The second axis presented a more fuzzy separation even though extreme samples were identified. Sample 35 was clearly characterised by a sulphur odour whereas samples 20 and 41 had a strong intensity of fat fish odour. The location of the other samples on this axis was mainly modulated by the intensities of these two descriptors. Dimension 3 of the PCA (not shown), created mainly by the descriptors brine note and potato odour added further information to discriminate samples 39, 38 and 14. A specific potato odour, associated with low dried and brine notes allowed these samples to be separated from the others.

Comparison of the two sensory maps

On the whole, the two procedures led to the same overall conclusion regarding product discrimination. The three-dimensional configurations obtained after running MDS on the sorting data and PCA on the profiling data led to an RV-coefficient equal to 0.81, i.e. good agreement between configurations. Whatever the sensory test used, the first dimension allowed the discrimination of the same two groups of products. One gathered fish hydrolysates with a dominant roasted odour while the second group, which showed a larger within-group variability, was constituted of products with odour characteristics other than the roasted note. The descriptors used in the sorting procedure to qualify this group were chemical, marine, crustacean, seaweed and sulphur odours while for the profiling test, fat fish, marine, rancid, sulphur and potato odours were used. In the two procedures, confidence ellipses for samples 20 and 41 were separated from those of samples 35, 37 and 40. However, only the profiling test highlighted the difference between sample 35 and products 37 and 40 and showed the specific potato odour of sample 39. Moreover, with the quantitative and descriptive analysis, it is possible to observe a gradient of intensity among samples with roasted note. Indeed, Fig. 2a shows different locations along the first axis for "roasted" 
products, in relation to the intensity attributed to this descriptor by the panellists. This information was completely masked in the sorting task; panellists sorted products according to the main odour characteristic, probably without taking into account its intensity. Nevertheless, although profiling could appear a more discriminative procedure, it is important to keep in mind that the step of descriptor selection is essential in the procedure. The example of sample 3 illustrates this point. This product was closer to the "roasted" group in the two configurations (sorting and profiling) but appeared significantly different from this group. If in sorting procedure the term "cheese" odour was the descriptor the most often used to qualify this product, in profiling, no descriptor allowed to identify this characteristic. Indeed, no similar product was present in the range of samples used during the attribute selection step and therefore this special characteristic of cheese odour was not identified. In this case, the sorting task gave more detailed information.

364

365

366

367

368

369

370

371

372

373

374

375

376

377

378

379

380

381

382

383

384

385

386

387

388

389

390

391

392

393

394

395

396

397

398

399

400

401

\subsection{Sensory map and relationship with volatile compounds}

The study of the relationships between sensory characteristics and volatile compounds was undertaken in order to find possible explanations for the description of hydrolysate odours..

In the case of the sorting procedure, the first dimension of the product configuration showed a high correlation with the group of volatile compounds identified as Maillard reaction products and of marine origin (Table 3) which probably explains the roasted note associated with this dimension. The main group of compounds correlated with dimension 2 was the fermentation origin group since the oxidation group was weakly linked to this dimension. This correlation was mainly due to sample 3, previously described by a cheese odour. The compounds identified in this sample were mainly alcohols (not shown). Regarding compounds from lipid oxidation, the best correlation was observed with the third dimension. For the two groups of hydrolysates identified on the first dimension, with and without sugar, a large distribution of the samples along dimension 3 can be noticed. A general trend of increasing lipid oxidation compounds from the bottom to the top of the dimension 3 was observed (not shown) and seems to explain this correlation.

With the profiling procedure, the first dimension of the product configuration showed the same correlation with Maillard and marine origin compounds as the sorting task as well as a correlation between compounds from fermentation and dimension 2. However, compounds from lipid oxidation did not show a clear correlation with any dimension. In this case, the discrimination between samples within the same group, i.e. with and without addition of sugar as described in the next subsection, was less clear and the relationships with compounds from lipid oxidation were weaker. This could be an effect of the profiling procedure; some descriptors, such as the roasted note, would be easier to detect and perhaps contribute to masking or to giving less importance to some attributes such as fat fish or rancid notes. In the profiling test, the distribution of samples according to roasted intensity, along dimension 1 , was clear for hydrolysates with sugar, and along dimension 2, according to fat fish intensity and sulphur odour, for hydrolysates without sugar. However, no common dimension enabled a simultaneous distribution of the two groups of products, as in sorting.

The study of the chemical structure of compounds did not add any more relevant information. Aldehydes, ketones, furans, acids and sulphurs were associated with compounds from the Maillard reaction and alcohols with a fermentation origin (data not shown) but further differences between the two procedures were not highlighted.

\subsection{Sensory map and relationship with processing parameters}

The effects of processing conditions on sensory characteristics were investigated using a quadratic model of regression on each of the dimensions obtained in MDS or PCA configurations. This model, previously used for the selection of products, involved the main 
effects, interaction effects and quadratic effects of these factors. This analysis also identified the significant effect by the $t$-values computed during the regression. These $t$ standardised values were represented on each of the configurations to highlight the main parameters involved in perceived sensory properties. For the sorting task, the first dimension illustrated the high effect of sugar as well as the quadratic effect of sugar (Fig. 3a). This variable explained the separation of samples into two groups along this axis, one with a strong roasted odour and the other without. The quadratic effect of sugar illustrated a non-linear relation in the perception of roasted odour. In fact, as previously described by Kouakou (2013), there was a significant increase in the roasted score between samples treated without sugar and those with $10 \mathrm{~g} \cdot \mathrm{Kg}^{-1}$ of sugar but this increase became smaller between 10 and $20 \mathrm{~g} . \mathrm{Kg}^{-1}$. The quadratic effect of hydrolysis time was mainly linked to dimensions 1 and 2 . A regression analysis of the roasted score only showed a clear optimum between 200 and 300 min of hydrolysis time for samples to which sugar had been added. The time-sugar interaction observed on dimension 2 could illustrate the effect of a long hydrolysis time on the odour of samples with sugar. Sample 3 is an example of these processing conditions where the longest hydrolysis time was applied on a sample with $10 \mathrm{~g} \cdot \mathrm{Kg}^{-1}$ of sugar. Dimension 3 of Fig. $3 \mathrm{~b}$ shows three main effects: hydrolysis time, hydrolysis temperature and the time-temperature interaction. High temperature and long hydrolysis time, without added sugar, led to samples with fat fish, chemical and marine odours (samples 20 and 41) while the interaction indicated that, for the same hydrolysis temperature, the choice of hydrolysis time modulated odours: for example, sample 40 was produced at $60^{\circ} \mathrm{C}$ for $30 \mathrm{~min}$ and presented sulphur, seaweed and spoilage characteristics whereas sample 41 was also hydrolysed at $60^{\circ} \mathrm{C}$ but for $360 \mathrm{~min}$ and its odour was qualified as marine and fat fish.

With the sensory profiling data, the regression of each dimension of the PCA on the design factors also showed highly significant linear and quadratic effects of sugar as well as a quadratic effect of hydrolysis time (Fig. 4a). The quadratic effect of time and the timetemperature interaction were also identified as significant effects $(p<0.10)$ (Fig. 4b). Compared to the results obtained from the sorting data, the temperature and the time-sugar interaction were not identified as factors with a significant explanatory power.

For the two sensory procedures, sorting task and profiling method, the linear and quadratic effects of sugar were identified as the most significant on hydrolysate sensory properties. It seems that the addition of sugar to hydrolysates led to the same global and dominant characteristic of roasted odour whatever the temperature or the time of hydrolysis. We can suppose that this major effect of sugar limits the analysis of other factor effects. However, it is possible for the two data sets to highlight the quadratic effect of hydrolysis time and the timetemperature interaction.

The sorting task data analysis pointed out a significant effect $(p<0.1)$ of hydrolysis timesugar interaction that was not revealed by profiling data. The assessment of sample 3 , prepared with sugar and corresponding to the highest level of time factor, probably explains this result. Moreover, this sample was better discriminated in the sorting task than in the profile test and therefore could contribute to identifying this significant effect. A temperature effect was also observed using the sorting data whereas it was not shown with the profiling data. We can suggest that the scoring of selected descriptors led panellists to recognise and score easily some criteria, such as the roasted note, and perhaps give less importance to the other attributes whereas panellists in the sorting task had more freedom in their assessment and could take into account the global perception of the sample without any influence of one particular characteristic. Dimension 3 (not presented) where the temperature effect was identified, confirmed for the two groups of samples, with or without a roasted note, a distribution of the products according to the temperature, high at the top and low at the bottom. It can also be noticed that sample 41 without enzyme stayed close to samples with 
enzymes, from a sensory point of view; this could suggest that an increase in the number of small peptides in the soluble phase under enzyme action would not significantly affect the global sensory characteristics of hydrolysates.

\section{Discussion and Conclusion}

From a methodological point of view, the results of this study confirm previous conclusions about the value of alternative procedures, such as free sorting, for sensory characterisation. Faye et al. (2004) showed that free sorting applied to the visual description of plastic pieces led to consistent data, a similarity of the sample configuration and the same conclusion about perception-process parameter relationships, compared to descriptive mapping. More recent works and reviews (Cartier et al. 2006, Varela and Ares 2012, Dehlhom et al. 2012, Nestrud and Lawless 2010) have emphasised the benefits of using alternative procedures, such as projective mapping, flash profiling and free sorting, which are generally faster and less timeconsuming than the classic descriptive and quantitative analysis while still producing meaningful product configurations, even with untrained panellists. Our study on odour characterisation illustrates this value and also shows that when descriptors are missing in the final list of attributes submitted to panellists, not having been identified during the selection step in profiling, free sorting can be more informative. In fact, the sorting task takes into account the whole space of products to find attributes and can therefore be an interesting step to describe sensory characteristics, even though some authors (Varela and Ares 2012, Chollet et al. 2011) have emphasized that the description by untrained panellists could be less detailed and sometimes more difficult to interpret. Varela and Ares (2012) also highlighted that descriptive analysis was more appropriate to identify small differences between products or to detect differences in intensity and therefore could not be replaced by these new techniques. The results of our case study agree with this fact. For example, panellists who scored the roasted note in salmon hydrolysates during profiling were able to discriminate the intensity of this odour while panellists who did free sorting were not.

From a practical point of view, the assessment of sensory characteristics by a free sorting task could be considered a relevant technique to obtain information for companies with no time to train a sensory panel and sufficient to identify the main sensory properties in product development. If more accurate information is needed, such as the intensity of the roasted odour in our study, it could easily be provided by a task like the ranking technique.

Regarding the use of a free sorting task for product development and the choice of process parameters, the results of this study on hydrolysates from salmon by-products show that this procedure can highlight process effects more easily than conventional profiling. The temperature effect observed using sorting data but not profiling data, as well as the better correlation between oxidation compounds and dimension 3 in the free sorting configuration, could suggest that a natural task of sorting without any fixed sensory vocabulary can offer more freedom in the panellist assessment and can take into account a global perception which sometimes allows more discrimination. The holistic approach of the sorting procedure shows the power of this tool based on a natural task of difference perception, which does not require any conscious evaluation or analytical quantification as in profiling.

However, although free sorting or a more sophisticated approach such as taxonomic free sorting can appear attractive in the industrial context of product development, the method seems less accurate for evaluating the intensity of sensory characteristics. Moreover, the number and characteristics of products to be assessed in the same session could be a restrictive factor. Some adaptations to these tests must be developed to allow a more general use. Nevertheless, this procedure is an attractive test which could be easily used in industrial applications, not only to obtain sensory characteristics but also to optimise a process. In the 
502 case of hydrolysate production from salmon by-products, the addition of sugar to modify and 503 mask fish odours has successfully been identified using this method.

\section{Acknowledgements}

507 This paper is dedicated to Christelle Kouakou, the main contributor to the data collection 508 during her $\mathrm{PhD}$ period who suddenly deceased before the publication. The authors want to 509 thank all the members of the two panels from Oniris and Ifremer, for their involvement in 510 sensory sessions. 
ABDI, H., VALENTIN, D., CHOLLET, S. and CHREA, C. 2007. Analyzing assessors and products in sorting tasks: DISTATIS, theory and applications. Food Qual. Prefer. 18(4), 627640. ATKINSON, A.C. AND DONEV, A.N. 1992. Optimum Experimental Design Oxford University Press, Oxford.

BLANCHER, G., CLAVIER, B.T., EGOROFF, C., DUINEVELD, K. and PARCON, J. 2011. A method to investigate the stability of a sorting map. Food Qual. Prefer. 23(1), 36-43. BORG, I. AND GROENEN, P.J.F. 2005. Modern multidimensional scaling. 2nd edition. Springer, New York.

CADORET, M., LÊ, S. and PAGÈS, J. 2009. A Factorial Approach for Sorting Task data (FAST). Food Qual. Prefer. 20(6), 410-417.

CADORET, M. and HUSSON, F. 2013. Construction and evaluation of confidence ellipses applied at sensory data. Food Qual. Prefer. 28, 106-115.

CARTIER, R., RYTZ, A., LECOMTE, A., POBLETE, F., KRYSTLIK, J., BELIN, E. and MARTIN, N. 2006. Sorting procedure as an alternative to quantitative descriptive analysis to obtain a product sensory map. Food Qual. Prefer. 17(7-8), 562-571.

CHOLLET, S., LELIÈVRE, M., ABDI, H. and VALENTIN, D. 2011. Sort and beer: Everything you wanted to know about the sorting task but did not dare to ask. Food Qual. Prefer. 22(6), 507-520.

COURCOUX, P., QANNARI, E.M., TAYLOR, Y., BUCK, D. and GREENHOFF, K. 2011. Taxonomic free sorting. Food Qual. Prefer. 23(1), 30-35.

DEHLHOLM, C., BROCKHOFF, P.B. and BREDIE, W.L.P. 2012. Confidence ellipses: A variation based on parametric bootstrapping applicable on Multiple Factor Analysis results for rapid graphical evaluation. Food Qual. Prefer. 26(2), 278-280.

FAYE, P., BRÉMAUD, D., DURAND DAUBIN, M., COURCOUX, P., GIBOREAU, A. and NICOD, H. 2004. Perceptive free sorting and verbalization tasks with naive subjects: an alternative to descriptive mappings. Food Qual. Prefer. 15(7-8), 781-791.

FAYE, P., BRÉMAUD, D., TEILLET, E., COURCOUX, P., GIBOREAU, A. and NICOD, H. 2006. An alternative to external preference mapping based on consumer perceptive mapping. Food Qual. Prefer. 17(7-8), 604-614.

FEDOROV, V.V. 1972. Theory of Optimal Experiments. Biometrika 59(3), 697-698 GIBOREAU, A., NAVARRO, S., FAYE, P. and DUMORTIER, J. 2001. Sensory evaluation of automotive fabrics: the contribution of categorization tasks and non verbal information to set-up a descriptive method of tactile properties. Food Qual. Prefer. 12(5-7), 311-322. ISO, International Organization for Standardization. 2003. Sensory Analysis-Methodology General Guidance for Establishing a Sensory Profile, ISO 13299, Geneva, Switzerland. KOUAKOU, C., BERGE, J.P., BARON , R., LETHUAUT, L., PROST, C. and CARDINAL, M. 2013. Odour modification in salmon hydrolysates using the Maillard reaction. J. Aquat. Food Prod. T. http://www.tandfonline.com/doi/abs/10.1080/10498850.2012.724153 LAWLESS, H.T., SHENG, N. and KNOOPS, S.S.C.P. 1995. Multidimensional scaling of sorting data applied to cheese perception. Food Qual. and Pref. 6(2), 91-98.

LELIËVRE, M., CHOLLET, S., ABDI, H. and VALENTIN, D. 2008. What is the validity of the sorting task for describing beers? A study using trained and untrained assessors. Food Qual. Prefer. 19(8), 697-703.

MORTON, J. 1969. Categories of interference: verbal mediation and conflict in card sorting. Brit. J. Psychol. 60 (3), 329-346.

NESTRUD, M.A. and LAWLESS, H.T. 2010. Perceptual mapping of apples and cheeses using projective mapping and sorting. J. Sensory Stud. 25, 390-405. 
563 ROBERT, P. and ESCOUFIER, Y. 1976. A unifying tool for linear multivariate statistical 564 methods: The RV-coefficient. Appl. Stat. 25(3), 257-265.

565 ROSCH, E. 1973. Natural categories. Cognitive Psychol. 7, 328-350.

566 SAINT-EVE, A., PAÇI KORA, E. and MARTIN, N. 2004. Impact of the olfactory quality 567 and chemical complexity of the flavouring agent on the texture of low fat stirred yogurts 568 assessed by three different sensory methodologies. Food Qual. Prefer. 15(7-8), 655-668.

569 SANTOSA, M., ABDI, H. and GUINARD, J.-X. 2010. A modified sorting task to investigate 570 consumer perceptions of extra virgin olive oils. Food Qual. Prefer. 21(7), 881-892.

571 STONE, H., SIDEL, J., OLIVER, S., WOOLSEY, A. and SINGLETON, R.C. 1974. Sensory 572 evaluation by quantitative descriptive analysis. Food Technol. 28, 24-34.

573 TANG, C. and HEYMANN, H. 2002. Multidimensional sorting, similarity scaling and free574 choice profiling of grape jellies. J. Sensory Stud. 17, 493-509.

575 VARELA, P. and ARES, G. 2012. Sensory profiling, the blurred line between sensory and 576 consumer science. A review of novel methods for product characterization. Food Res. Int. 48, 577 893-908.

578 VARELA, P., ARES, G. and FISZMAN, S. 2013. Texture and semantics: the conceptual 579 structure in consumers' minds. J. Sensory Studies. 28(3), 194-204.

580 VERAMENDI, M., HERENCIA, P. and ARES, G. 2013. Perfume odor categorization: to 581 what extent trained assessors and consumers agree? J. Sensory Studies. 28, 76-89.

582 WILD, C., SINGER, M., ROSMAN, B., RICCI, J., LIDZ, T. 1965. Measuring disordered 583 styles of thinking. Using the Object Sorting Test on parents of schizophrenic patients. Gen. 584 Psychiat. 13(5), 471-476. 
Table 1 Identification of the 21 experimental points and corresponding process variables, selected from the initial experimental design

Table 2 Terms used by panellists to describe samples of salmon hydrolysates associated to a 595 group in the sorting task

Table 3 Pearson Correlations between volatile compounds classified according to their origin group and each dimension of the product configuration for the sorting task and profiling 599 procedures 
600

$601 \quad$ Table 1

602

\begin{tabular}{|c|c|c|c|c|}
\hline $\begin{array}{l}\text { Process variables } \\
\text { Sample code }\end{array}$ & $\begin{array}{l}\text { Time } \\
(\mathrm{min})\end{array}$ & $\begin{array}{c}\text { Temperature } \\
\left({ }^{\circ} \mathrm{C}\right)\end{array}$ & $\begin{array}{c}\text { Antioxidant } \\
\text { (tocopherol and } \\
\text { rosemary) (ppm) }\end{array}$ & $\begin{array}{c}\text { Sugar } \\
\text { (xylose) } \\
\left(\text { g.kg }{ }^{-1}\right)\end{array}$ \\
\hline 2 & 30 & 45 & 125 & 10 \\
\hline 3 & 470 & 45 & 125 & 10 \\
\hline 6 & 140 & 60 & 125 & 10 \\
\hline 8 & 140 & 40 & 0 & 10 \\
\hline 9 & 360 & 50 & 250 & 10 \\
\hline 11 & 250 & 35 & 250 & 10 \\
\hline 13 & 250 & 55 & 0 & 10 \\
\hline 14 & 140 & 40 & 93.75 & 0 \\
\hline 15 & 360 & 50 & 156.25 & 20 \\
\hline 16 & 140 & 50 & 156.25 & 20 \\
\hline 17 & 250 & 35 & 156.25 & 20 \\
\hline 18 & 250 & 45 & 31.25 & 20 \\
\hline 20 & 250 & 55 & 93.75 & 0 \\
\hline 27 & 360 & 50 & 156.25 & 20 \\
\hline 33 & 250 & 45 & 0 & 0 \\
\hline 35 & 30 & 30 & 0 & 0 \\
\hline 37 & 30 & 60 & 250 & 0 \\
\hline 38 & 360 & 30 & 250 & 0 \\
\hline 39 & 360 & 30 & 0 & 0 \\
\hline 40 & 30 & 60 & 0 & 0 \\
\hline 41 & 360 & 60 & 0 & 0 \\
\hline
\end{tabular}

603

604 
606

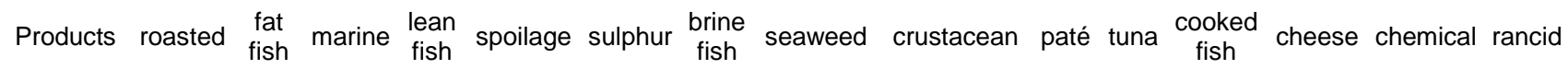

\begin{tabular}{cccccccccccccccc}
\hline 2 & 13 & 11 & 4 & 1 & 2 & 0 & 5 & 4 & 3 & 3 & 4 & 2 & 0 & 0 & 0 \\
3 & 8 & 5 & 3 & 1 & 8 & 4 & 3 & 0 & 1 & 5 & 1 & 3 & 10 & 0 & 1 \\
6 & 12 & 12 & 4 & 1 & 2 & 0 & 5 & 2 & 3 & 4 & 4 & 1 & 0 & 1 & 0 \\
8 & 13 & 10 & 7 & 1 & 2 & 0 & 5 & 4 & 1 & 4 & 4 & 2 & 0 & 0 & 0 \\
9 & 13 & 11 & 6 & 1 & 4 & 0 & 7 & 2 & 2 & 2 & 3 & 2 & 0 & 0 & 0 \\
11 & 15 & 7 & 8 & 2 & 3 & 2 & 2 & 3 & 1 & 5 & 2 & 2 & 1 & 1 & 0 \\
13 & 11 & 13 & 5 & 5 & 4 & 0 & 4 & 1 & 2 & 2 & 2 & 4 & 0 & 1 & 0 \\
14 & 3 & 9 & 7 & 7 & 3 & 10 & 1 & 4 & 2 & 0 & 0 & 1 & 2 & 2 & 1 \\
15 & 18 & 9 & 6 & 2 & 5 & 1 & 5 & 0 & 3 & 2 & 1 & 2 & 1 & 0 & 0 \\
16 & 14 & 7 & 4 & 2 & 4 & 1 & 3 & 3 & 2 & 5 & 5 & 3 & 0 & 1 & 1 \\
17 & 13 & 11 & 4 & 3 & 0 & 1 & 8 & 0 & 2 & 6 & 4 & 3 & 1 & 1 & 0 \\
18 & 13 & 7 & 6 & 2 & 2 & 1 & 5 & 2 & 2 & 3 & 3 & 2 & 1 & 0 & 0 \\
20 & 3 & 6 & 8 & 7 & 4 & 4 & 2 & 3 & 4 & 0 & 1 & 3 & 1 & 4 & 0 \\
27 & 14 & 9 & 8 & 2 & 4 & 2 & 7 & 0 & 3 & 2 & 1 & 4 & 0 & 1 & 1 \\
33 & 3 & 12 & 8 & 9 & 4 & 2 & 1 & 5 & 4 & 0 & 1 & 2 & 1 & 1 & 2 \\
35 & 0 & 2 & 6 & 8 & 5 & 16 & 0 & 7 & 6 & 0 & 1 & 0 & 3 & 0 & 0 \\
37 & 1 & 4 & 6 & 7 & 4 & 11 & 3 & 7 & 4 & 0 & 1 & 1 & 3 & 2 & 0 \\
38 & 4 & 11 & 7 & 6 & 6 & 1 & 4 & 4 & 4 & 0 & 1 & 2 & 0 & 3 & 2 \\
39 & 4 & 2 & 11 & 4 & 4 & 4 & 2 & 5 & 7 & 1 & 1 & 0 & 1 & 3 & 1 \\
40 & 1 & 8 & 3 & 4 & 8 & 13 & 0 & 8 & 4 & 0 & 0 & 1 & 1 & 0 & 0 \\
41 & 0 & 6 & 9 & 5 & 2 & 5 & 3 & 7 & 7 & 0 & 1 & 1 & 0 & 3 & 4 \\
\hline total & 176 & 172 & 130 & 80 & 80 & 78 & 75 & 71 & 67 & 44 & 41 & 41 & 26 & 24 & 13
\end{tabular}

607

608

609

610

611

612

613

614

615

616

Table 3

\begin{tabular}{lllllll}
\hline & \multicolumn{3}{c}{ Sorting procedure } & \multicolumn{3}{c}{ Profiling procedure } \\
\multicolumn{1}{c}{ Origin } & Dim1 & Dim2 & Dim3 & Dim1 & $\operatorname{Dim} 2$ & $\operatorname{Dim} 3$ \\
\hline Lipid oxidation & 0.15 & -0.36 & $\mathbf{0 . 5 3}$ & 0.19 & 0.10 & 0.18 \\
Maillard reactions & $(0.50)$ & $(0.11)$ & $\mathbf{( 0 . 0 1 )}$ & $(0.41)$ & $(0.66)$ & $(0.44)$ \\
& $\mathbf{0 . 6 9}$ & -0.14 & -0.29 & $\mathbf{0 . 7 7}$ & -0.009 & 0.19 \\
Fermentation & $\mathbf{( 0 . 0 0 0 6 )}$ & $(0.53)$ & $(0.20)$ & $\mathbf{( 0 . 0 0 0 0})$ & $(0.97)$ & $(0.40)$ \\
& 0.34 & $\mathbf{- 0 . 5 1}$ & 0.19 & 0.30 & -0.30 & 0.05 \\
Marine & $(0.13)$ & $\mathbf{( 0 . 0 2})$ & $(0.40)$ & $(0.18)$ & $(0.19)$ & $(0.83)$ \\
& $\mathbf{0 . 6 2}$ & 0.19 & 0.07 & $\mathbf{0 . 7 1}$ & 0.03 & -0.0001 \\
Other & $(\mathbf{0 . 0 0 3})$ & $(0.40)$ & $(0.75)$ & $\mathbf{( 0 . 0 0 0 3 )}$ & $(0.90)$ & $(0.99)$ \\
& -0.19 & -0.03 & 0.03 & -0.05 & 0.10 & 0.16 \\
& $(0.39)$ & $(0.88)$ & $(0.20)$ & $(0.82)$ & $(0.67)$ & $(0.50)$ \\
\hline
\end{tabular}

In brackets, significant level of the correlation 
Figure captions

FIG. 1a. SORTING DATA - REPRESENTATION OF FISH HYDROLYSATES WITH 90\% CONFIDENCE ELLIPSES ON THE BASIS OF THE FIRST TWO MDS DIMENSIONS Dimension 1 explains $60.9 \%$ of the variation and dimension 2 explains $20.3 \%$ of the variation

FIG. 1b. SORTING DATA - CORRELATION OF THE DESCRIPTION TERMS WITH THE FIRST TWO MDS DIMENSIONS

FIG. 2a. PROFILING DATA - REPRESENTATION OF FISH HYDROLYSATES WITH 90\% CONFIDENCE ELLIPSES ON THE BASIS OF THE FIRST TWO DIMENSIONS OF PCA. Dimension 1 explains $75.1 \%$ of the variation and dimension 2 explains $10.25 \%$ of the variation

FIG. 2b. PROFILING DATA - PROJECTION OF DESCRIPTORS IN THE FIRST PLANE OF PCA

FIG. 3. SORTING DATA - REPRESENTATION ON THE DIMENSIONS OF MDS OF THE T-VALUES OF THE PROCESSING PARAMETERS FROM A QUADRATIC MODEL REGRESSION
(a) first two dimensions, (b) dimensions 1-3

FIG. 4. PROFILING DATA - REPRESENTATION ON THE PRINCIPAL COMPONENTS OF PCA OF THE T-VALUES OF THE PROCESSING PARAMETERS FROM A QUADRATIC MODEL REGRESSION
(a) first two dimensions, (b) dimensions 1-3 
652 FIG. 1a. SORTING DATA - REPRESENTATION OF FISH HYDROLYSATES WITH 90\%

Fig. 1a

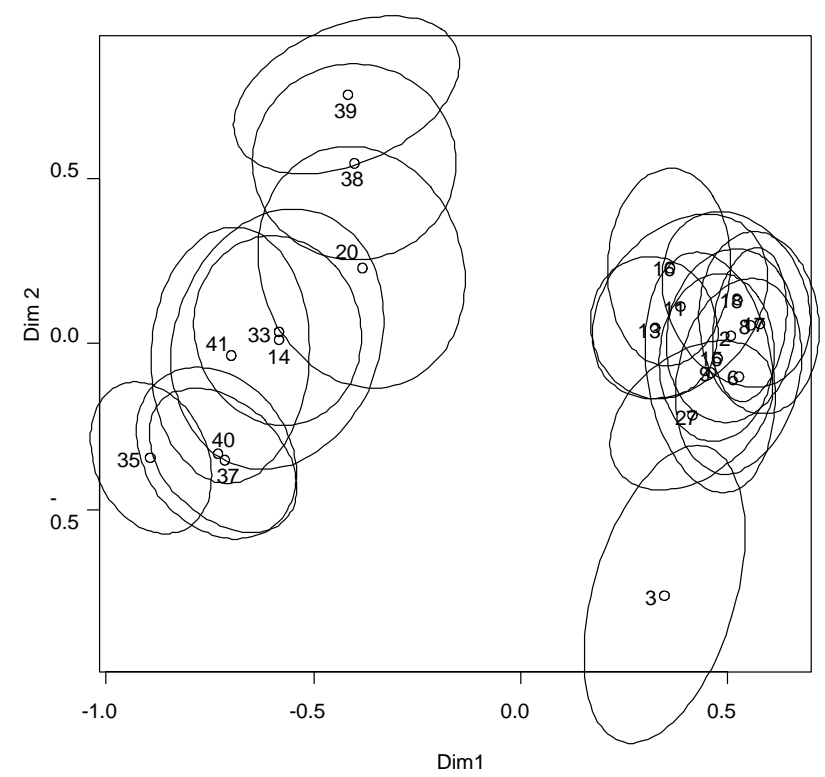

Fig. 1b

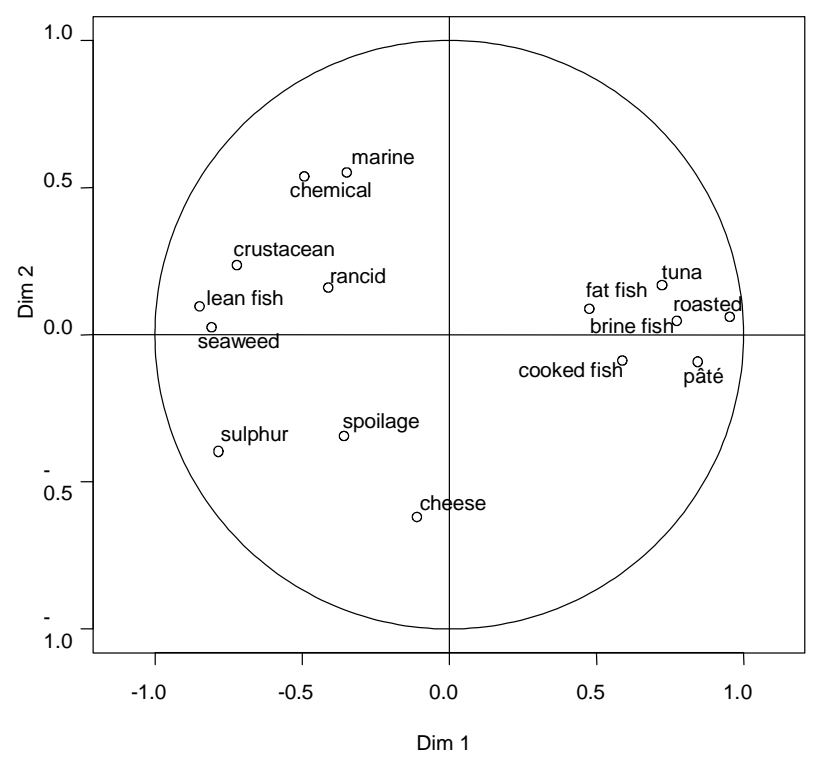


663

664

665

666

667

668

669

670

671

672

673
Fig. 2b

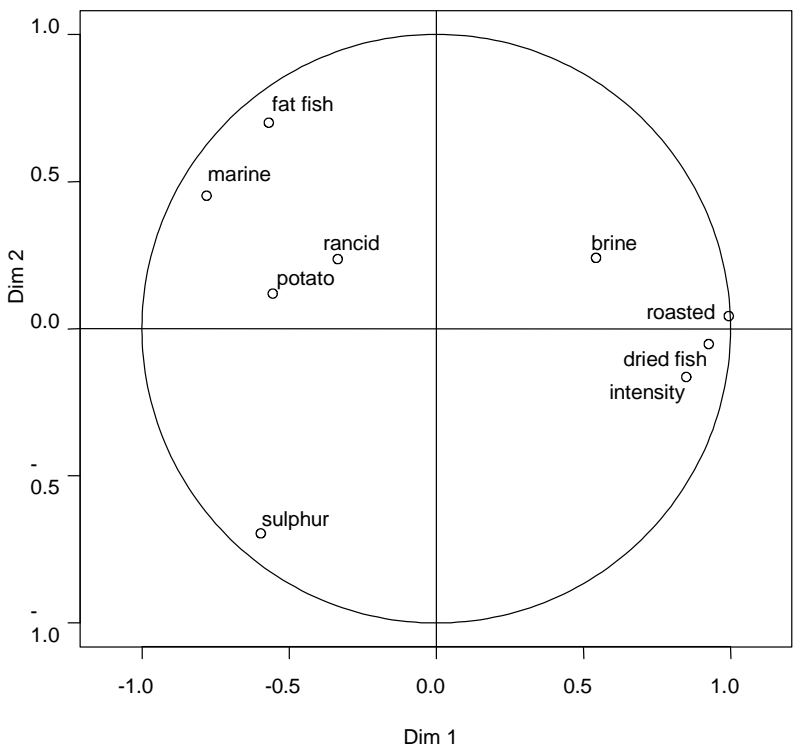

674

675

676

677

678

679

680
FIG. 2a. PROFILING DATA - REPRESENTATION OF FISH HYDROLYSATES WITH 90\% CONFIDENCE ELLIPSES ON THE BASIS OF THE FIRST TWO DIMENSIONS OF PCA. Dimension 1 explains $75.1 \%$ of the variation and dimension 2 explains $10.25 \%$ of the variation

FIG. 2b. PROFILING DATA - PROJECTION OF DESCRIPTORS IN THE FIRST PLANE OF PCA

Fig. 2a

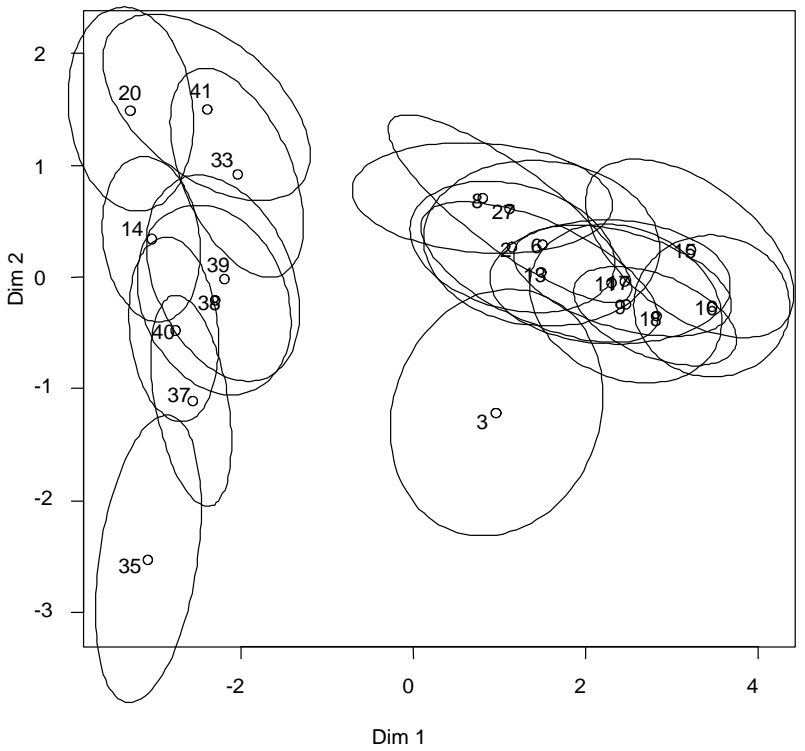


FIG. 3. SORTING DATA - REPRESENTATION ON THE DIMENSIONS OF MDS OF

(a) first two dimensions, (b) dimensions 1-3

685

686

687

688

689

690

(a)

(b)

691
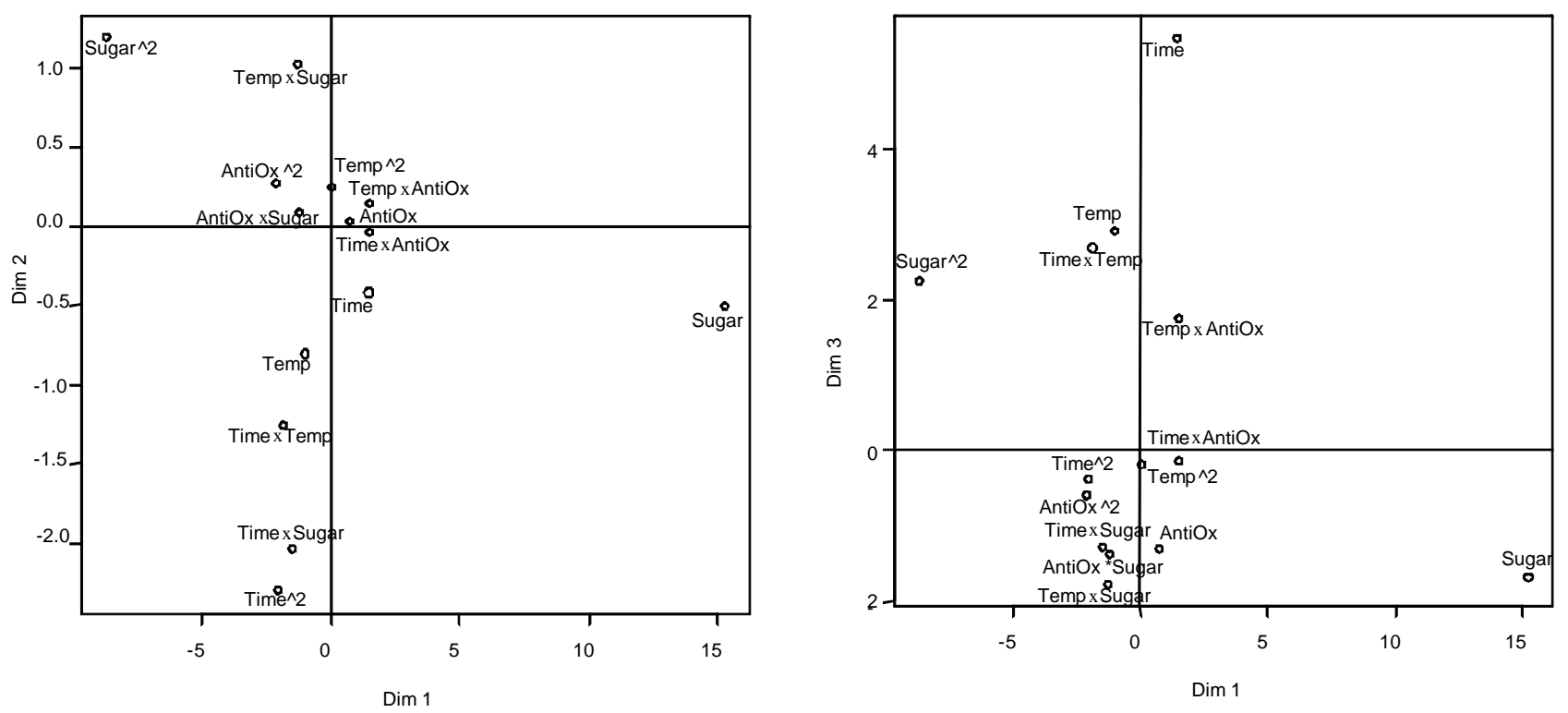

692

693

694

695

696 
FIG. 4. PROFILING DATA - REPRESENTATION ON THE PRINCIPAL COMPONENTS OF PCA OF THE T-VALUES OF THE PROCESSING PARAMETERS FROM A

700 QUADRATIC MODEL REGRESSION

701

(a) first two dimensions, (b) dimensions 1-3

702

703

704

705

706

(a)

(b)
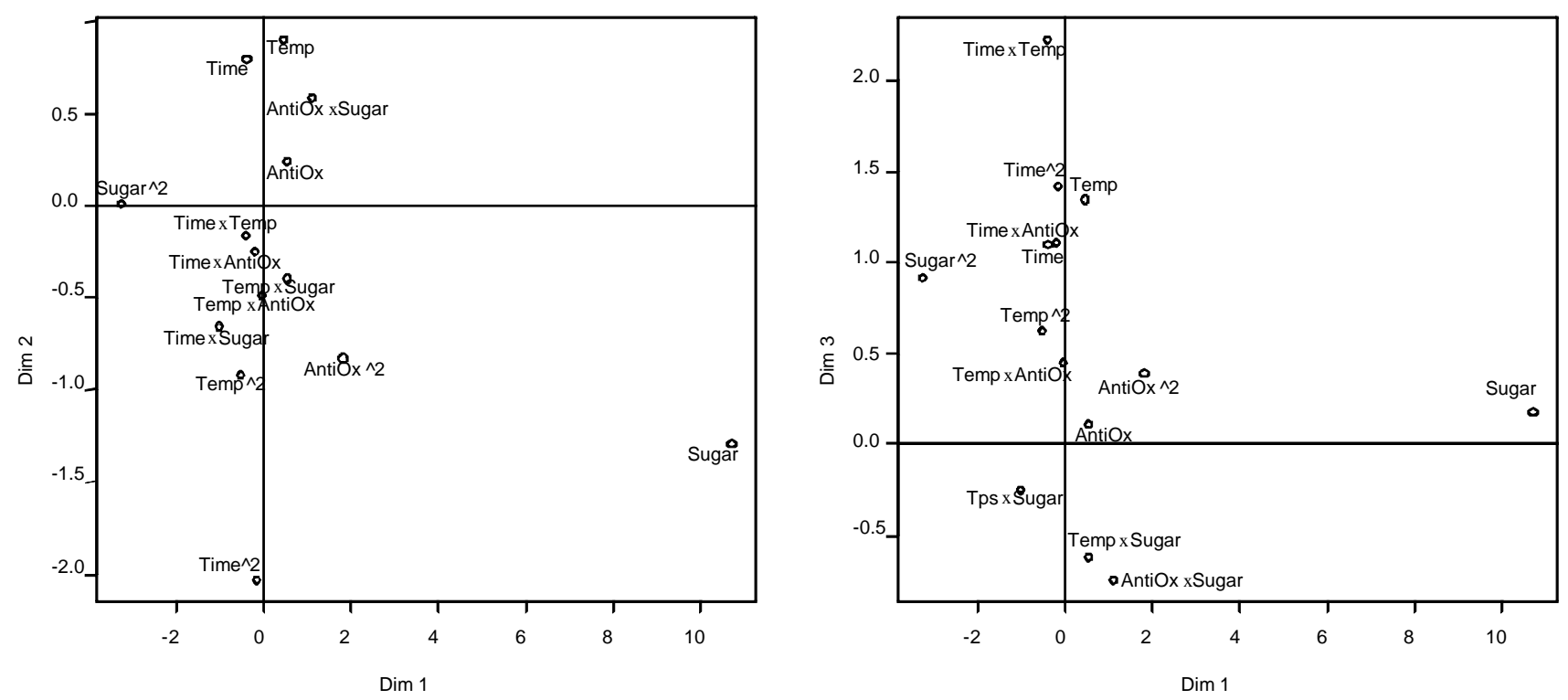